\title{
„Orientierende Untersuchung Urin" Warum streicht mir die KV die GOP 32 030?
}

Dr. med. K. N., Fachärztin für Allgemeinmedizin, KV Bayern:

Die KV streicht im Rahmen der sachlichen und rechnerischen Richtigstellung immer wieder die GOP 32 030, „,Orientierende Untersuchung Urin“. Ist dies korrekt? Antwort: Der Leistungsinhalt der GOP 32030 lautet: „Obligater Leistungsinhalt: Orientierende Untersuchung mit visueller Auswertung mittels vorgefertigter Reagenzträger oder Reagenzzubereitungen. Fakultativer Leistungsinhalt: Apparative Auswertung, Verwendung von Mehrfachreagenzträgern, $€ 0,50$. Können mehrere Bestandteile eines Körpermaterials sowohl durch Verwendung eines Mehrfachreagenzträgers als auch durch Verwendung mehre- rer Einfachreagenzträger erfasst werden, so ist in jedem Fall nur einmal die GOP 32030 berechnungsfähig.

Bei mehrfacher Berechnung der Gebührenordnungsposition 32030 ist die Art der Untersuchungen anzugeben. Die GOP ist nicht neben den GOP 01732 und 32880 bis 32882 berechnungsfähig."

Damit wäre eigentlich jede Urinuntersuchung bis auf die angegebenen Ausschlüsse abrechenbar, wäre da nicht die Präambel des Kapitels 32.2.1 Basisuntersuchungen: „Der Nachweis von Eiweiß und/oder Glukose im Harn (ggf. einschl. Kontrolle auf Ascorbinsäure) sowie die Bestimmung des spezifischen Gewichts und/oder des pH-Wertes im Harn ist nicht berechnungsfähig."
Damit der Vertragsarzt nicht ganz auf den Kosten sitzen bleibt, können die Teststreifen für den Nachweis von Eiweiß, Glukose, ggf. einschließlich Kontrolle auf Ascorbinsäure in vielen KVen über Sprechstundenbedarf bezogen werden.

Geht die Untersuchung über den definierten oder über die bei Vorsorgeuntersuchungen vorgesehenen Parameter hinaus, muss dies begründet werden, wenn die Untersuchung dennoch nicht mit einem Teststreifen durchgeführt werden kann. Die Kosten eines „erweiterten" Teststreifens gehen dann dennoch zu Lasten der Praxis. Erst ein zweiter Teststreifen geht zu Lasten der GKV.

\section{Telefonische Beratung im Notfalldienst}

\section{Wie komme ich an die Kassengebühr?}

Frau Dr. S. S., Ärztin für Allgemeinmedizin, KVB:

Im Rahmen des Vertragsärztlichen Notfalldienstes (NFD) erbringe ich immer wieder telefonische Beratungen. Die eventuell unvollständigen Daten erfragen meine Mitarbeiter beim vertretenen Kollegen. In vielen Fällen muss Kassengebühr gezahlt werden. Wie komme ich an das Geld?

Antwort: Hier gibt es leider kein Patentrezept. Die örtlichen Gegebenheiten spielen hier eine große Rolle. Eines ist sicher: Auch für eine telefonische Beratung im Rahmen des NFD fällt zusätzlich zur Quartalsgebühr eine Kassengebühr an. Nur wenn der Patient im laufenden Quartal bereits einmal die Kassengebühr für den NFD gezahlt hat oder von der Zuzahlungspflicht insgesamt befreit ist, muss er nicht noch einmal zahlen. Beide Voraussetzungen muss der Patient entweder durch Vorlage der Quittung oder der Befreiungsbescheinigung nachweisen. Dazu muss er entweder persönlich in die Praxis kommen oder er schickt ein Fax mit einer Kopie oder eine Mail mit entsprechendem Anhang. Solange er diesen Nachweis nicht führt, ist er zahlungspflichtig. Die Daten für eine Überweisung der zehn Euro können ebenfalls per Fax oder Mail übermittelt werden.

Bezahlt der Patient nicht, läuft das übliche Verfahren: Er bekommt per Brief das vorgesehene Mahnschreiben. Die Portogebühr wird verrechnet mit der GOP 80 046. Diesem fügen wir einen vorgefertigten Überweisungsträger für die Kassengebühr wie bei Privatliquidationen bei. Geht bis zur Quar- talsabrechnung keine Kassengebühr ein, wird der Patient mit der entsprechenden GOP 80 044, „Praxisgebühr trotz Mahnung nicht erhoben", gekennzeichnet. Damit ist für den Vertragsarzt die Angelegenheit erledigt. Es ist dann Sache der Kassenärztlichen Vereinigung, den säumigen Patienten erneut zu mahnen und gegebenenfalls ein gebührenpflichtiges Inkasso einzuleiten. Verfahren Sie nicht so, wird die KV die Gebühr im Rahmen der Honorarberechnung einbehalten. Dies geschieht automatisch, wenn keine der GOP angesetzt werden kann, die für den Nachweis eines Befreiungsgrundes stehen. 